\title{
Patterns of genetic diversity and population structure of the threatened Houbara and Macqueen's bustards as revealed by microsatellite markers
}

\author{
A. Korrida ${ }^{1,2}$, S. Jadallah ${ }^{1}$, F. Chbel ${ }^{3}$, A. Amin-Alami ${ }^{2}$, M. Ahra ${ }^{2}$ and S. Aggrey ${ }^{4}$ \\ ${ }^{1}$ Genetics Laboratory, HRH Prince Sultan Bin Abdul Aziz A1 Saud, \\ International Foundation for Conservation and Development of Wildlife, \\ Agadir, Morocco \\ ${ }^{2}$ Laboratoire des Substances Naturelles, Faculté des Sciences d'Agadir, \\ Université Ibn Zohr, Agadir, Morocco \\ ${ }^{3}$ Laboratoire National de Police Scientifique, Service Biologie, \\ Quartier Palmier, Mâarif, Casablanca, Morocco \\ ${ }^{4}$ Department of Poultry Science, University of Georgia, Athens, GA, USA \\ Corresponding author: A. Korrida \\ E-mail: amalkorrida11@aol.com \\ Genet. Mol. Res. 11 (3): 3207-3221 (2012) \\ Received June 15, 2011 \\ Accepted June 20, 2012 \\ Published September 12, 2012 \\ DOI http://dx.doi.org/10.4238/2012.September.12.4
}

\begin{abstract}
The Houbara bustard (Chlamydotis undulata) is a threatened avian species that is rapidly declining throughout its range, especially in North Africa, Asia and the Canary Islands. We examined the population structure and genetic variation for the three Houbara subspecies C. undulata undulata, $C$. $u$. fuertaventurae and $C . u$. macqueenii. A total of 266 birds from 10 populations were genotyped using seven polymorphic microsatellite markers. The analysis of microsatellite loci generated 1821 genotypes and 55 different alleles. Estimates of observed and expected heterozygosities were relatively high and ranged from 0.371 to 0.687 and from 0.326 to 0.729 , respectively. For the first time, significant phylogeographic structure among Asian
\end{abstract}


Houbara populations was found using neutral nuclear markers. Analysis of molecular variance revealed $12.03 \%$ population variability among the subspecies. Population structure and assignment tests inferred using a Bayesian approach revealed two distinct clusters with more than $90 \%$ likelihood, one Asian and one North African. A positive correlation between genetic distance and geographic distance was detected among populations $\left(\mathrm{r}^{2}=0.302\right)$. For conservation purposes, this genetic information will help understand the current genetic status improving management strategies for Houbara bustard breeds and populations.

Key words: Microsatellite markers; Houbara bustard; Genetic diversity; Conservation genetics

\section{INTRODUCTION}

Houbara bustard (Chlamydotis undulata) is an avian polytypic species with habitats in arid and semi-arid environments characterized by lower and middle altitudes, steppes, sandy, and stony wadis with shrubby halophytic vegetation (Cramp and Simmons, 1980). The species belongs to the Otididae family made up of 23 species and is divided into three subspecies according to geographic distribution and morphology. C. u. undulata (Cuu) (Jacquin, 1784), found in Northern Africa (north Mauritania, Morocco, Algeria to Egypt) is sedentary, rarely vagrant or locally nomadic (Heim de Balsac and Mayaud, 1962). C. $u$. macqueenii (Cum) (Gray, 1832), the migratory form of "Chlamydotis" genus is found in Asia (from the Middle East to Altai) and lives in Sinai, Arabia, Baluchistan, Jordan, north Caspian Sea, north-west India, Afghanistan, Pakistan, Kazakhstan, Iran, Iraq, and Mongolia. C. u. fuertaventurae (Cuf) (Rothschild and Hartert, 1894), a non-migratory and endemic subspecies to the Canary Islands resides mainly in Fuerteventura and Lanzarote.

The taxonomic status of the Chlamydotis genus remains controversial to this day. According to Sangster et al. (1999), Knox et al. (2002) and Lesobre et al. (2010), the Chlamydotis genus consists of two species $C$. undulata and $C$. macqueenii with $C$. undulata comprising two subspecies ( $\mathrm{Cuu}$ and $\mathrm{Cuf}$ ). Other authors, however, posit that the genus is monotypic and made up of three well-differentiated subpopulations [Cramp and Simmons, 1980; Pitra et al., 2002, 2004; Idaghdour et al., 2004; IUCN, 2010; Birdlife International (2010) taxonomic group]. In this paper, we opt for the latter classification. According to the red list of threatened species (IUCN, 2010), Houbara bustard is classified "vulnerable". The ongoing decrease in the size of the Houbara bustard populations requires that knowledge of the genetic structure, population boundaries and level of connectivity between populations be known for proper management (Haddane, 1985). Furthermore, genetic drift ultimately affects gene flow and as stated by Wright (1951), gene flow may allow the spread of favorable adaptation among populations. Thus, in a species like $C$. undulata divided into populations, gene flow is likely to help the species overcome problems related to loss of adaptive genetic diversity (Keller et al., 1994). Estimates of gene flow between island and mainland Houbara populations or among mainland populations can provide useful information for management initiatives aiming for the long-term persistence and conservation of the species in its natural habitat. In Morocco, the Houbara bustard popula- 
tion has declined substantially as a result of overgrazing, habitat destruction and excessive hunting (Birdlife International, 2010). Assessing genetic diversity and population structure of wildlife species using neutral molecular markers allows determination of their extinction risk and to design strategies for their management and conservation (Serrano et al., 2009). Previous genetic studies on Houbara bustards have failed to show an evidence for genetic differentiation between the North African C. undulata. Idaghdour et al. (2004) using mtDNA showed extensive structuring among the three subspecies but could not find structure within Cuu. Dawson et al. (1999) while performing analysis on the CHD-Z gene, confirmed the absence of size difference products between two Houbara bustard populations from Morocco and the Canary Islands. Lesobre et al. (2010) used both mtDNA and DNA microsatellites as described in Idaghdour et al. (2004) and Chbel et al. (2002), respectively, and did not find solid evidence of structuring within Cuu either. Pitra et al. (2004) studied the macqueenii population history and showed weak structuring using only four microsatellite markers.

The objective of this research was to implement Chbel et al. (2002) polymorphic SSR markers and extend their use into a larger number of populations in order to pertinently evaluate and assess genetic distance, phylogenetic relationships, genetic diversity, and population differentiation between the North African forms $\mathrm{Cuu}$ and $\mathrm{Cuf}$ and the Asian form Cum.

\section{MATERIAL AND METHODS}

The isolation of single sequence repeats (SSR) loci was carried out from Moroccan Houbara's extracted DNA. Library construction and primer design were done by Genetic Identification Service Inc. (USA) as described in Chbel et al. (2002). From 1993 to 2007, the three Houbara bustard subspecies were sampled from the 10 different locations listed in Figure 1 and Table 1 avoiding the sampling of close related individuals. A total of $135 \mathrm{blood}$ samples were collected from the Cuu founding stock at IFCDW originated from Erfoud, Errachidia and Boudnib areas where each sample represents an egg from a single clutch. Another 18 tissue samples were obtained from hunted birds in Laayoune region in south of Morocco. The Cum collection included five different populations. Blood and tissue samples from Iran $(\mathrm{N}=16)$, Jordan $(\mathrm{N}=2)$ and Baluchistan $(\mathrm{N}=28)$ populations were obtained from the National Wildlife Research Center (NWRC) in Taif, Kingdom of Saudi Arabia and samples from the Negev desert $(\mathrm{N}=3)$, Afghanistan $(\mathrm{N}=6)$ and mixed origins of the Asiatic Houbara from Kazakhstan, Saudi Arabia and Pakistan were collected from Sheikh Butti Maktoum's Wildlife Center in the United Arab Emirates. We later labeled samples from Jordan and the Negev desert as the Middle East sample. For Cuf, we received 21 blood samples from La Oliva Breeding Station in the Canary Islands. Blood samples were stored in $\mathrm{ACD}(0.48 \%$ citric acid, $1.32 \%$ sodium citrate and $1.47 \%$ dextrose) or QLB ( $1 \%$ sodium $\mathrm{N}$-lauryl sarcosine, $10 \mathrm{mM}$ EDTA, $10 \mathrm{mM} \mathrm{NaCl}$ and $10 \mathrm{mM}$ Tris, $\mathrm{pH}$ 8.0) buffers at either $-20^{\circ} \mathrm{C}$ or room temperature whereas tissue samples in a saline lysis buffer $(20 \% \mathrm{DMSO}$ and $5 \mathrm{mM} \mathrm{NaCl}$ ) and stored in ambient temperature.

Total genomic DNA was extracted using either phenol-chloroform method (Sambrook et al., 1989), GEN-IAL First DNA, or QIAmp DNA Kits. DNA concentration was quantified using Hoefer DyNA Quant 200 Fluorometer and its integrity checked on 1\% agarose gels. Gradient PCRs were conducted during the optimization phase using Biometra T1 and Techne 
GeneE thermal cyclers. In a final volume of $5 \mu \mathrm{L}$, the PCR master mix contained: $10 \mathrm{X}$ Promega buffer, $\mathrm{dH}_{2} \mathrm{O}, 25 \mathrm{mM} \mathrm{MgCl}, 2 \mathrm{mM}$ dNTPs, $20 \mu \mathrm{M}$ forward and reverse primers, $1 \mu \mathrm{M}$ IRD-M13 forward primer (Schuelke, 2000), $5 \mathrm{U} / \mu \mathrm{L}$ Taq Polymerase Enzyme (Promega) and $20 \mathrm{ng}$ template DNA. PCRs were carried out with the following sittings: $94^{\circ} \mathrm{C}$ for $3 \mathrm{~min}$ for one cycle; $94^{\circ} \mathrm{C}$ for $20 \mathrm{~s}$, Tal for $1 \mathrm{~min}$ and $72^{\circ} \mathrm{C}$ for $30 \mathrm{~s}$ for 14 cycles; $94^{\circ} \mathrm{C}$ for $20 \mathrm{~s}$, Ta2 for $1 \mathrm{~min}$ and $72^{\circ} \mathrm{C}$ for $30 \mathrm{~s}$ for 28 cycles, and $72^{\circ} \mathrm{C}$ for $10 \mathrm{~min}$ for one final cycle. Annealing temperatures for all SSR markers were as in Chbel et al. (2002). PCR products were run and visualized on a Li-COR $4000 \mathrm{~L}$ sequencing device through a $6.5 \%$ Li-COR KB Plus Gel Matrix and microsatellite allele sizes were determined using an Infra Red Dye 800 size marker.

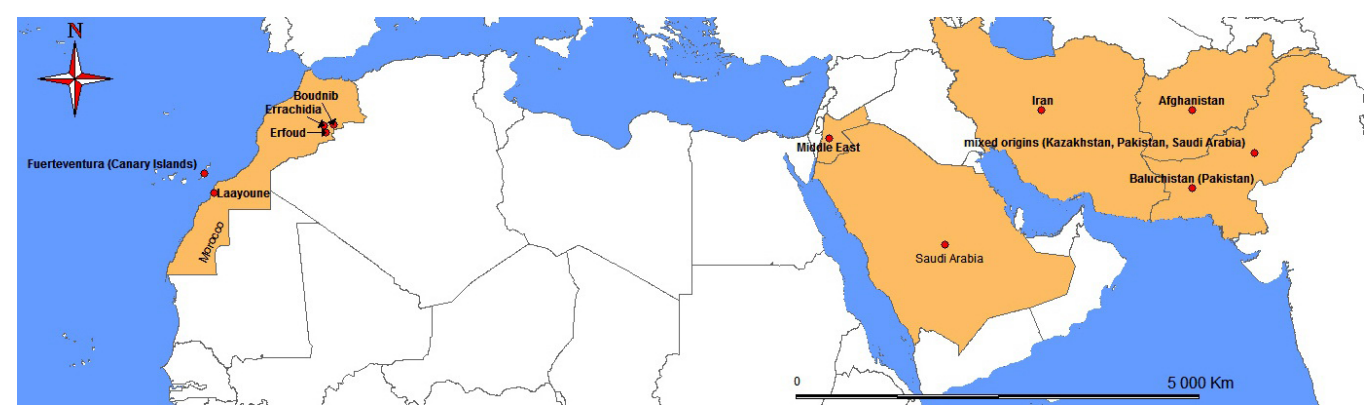

Figure 1. Locations of the 10 sampled populations of Houbara bustards.

\section{Data analysis}

Prior to data analysis, the Micro-Checker 2.2.3 program (Van Oosterhout et al., 2004) was used to identify microsatellite profiling errors. Tests for linkage disequilibrium were performed in GENEPOP 4.0 (Raymond and Rousset, 1995) and the Arlequin 3.1 software (Excoffier et al., 2006). The expected $\left(H_{\mathrm{E}}\right)$ and observed heterozygosities $\left(H_{\mathrm{O}}\right)$ for each population were estimated using the GENETIX 4.03 software (Belkhir et al., 2003) and the polymorphic information content (PIC) was estimated using the Cervus 2.0 program (Marshall et al., 1998). For each population, deviation from Hardy-Weinberg equilibrium (HWE) was tested with the Fisher exact test implemented in GENEPOP based on 5000 iterations and 20 batches. Bonferroni's adjustment (Rice, 1989) was implemented to compensate for multiple comparisons at $\mathrm{P}<0.05$ level. The analysis of molecular variance (AMOVA) describing the hierarchical partitioning of genetic variance among and within populations was carried out using the Arlequin package. By defining each time the number of groups considered in the structure, the AMOVA used permutation tests to evaluate null hypotheses of no population structure within groups and between groups. The $F_{\mathrm{ST}}$ index was also tested by permuting haplotypes among populations among groups and the $F_{\text {CT }}$ by permuting populations among groups. Population pairwise $F_{\mathrm{ST}}$ values were obtained for all population pairs and their significance values were tested against the assumption of no difference between populations by considering 1000 random permutations of haplotypes. The Arlequin package was also utilized to assess the genetic differentiation $\left(F_{\mathrm{ST}}\right.$; P values) for all population pairwise combinations considering the number of different alleles as the molecular distance. The Genetic 
Data Analysis program (Lewis and Zaykin, 2001) was used to calculate Wright's fixation indices $F_{\mathrm{IS}}, F_{\mathrm{ST}}$ and $F_{\mathrm{IT}}$ (Wright, 1951) according to Weir and Cockerham (1984) and using the Jackniffing approach over populations. The MEGA 4.1 software (Tamura et al., 2007) was used to construct the minimum evolution (ME) tree (Nei et al., 1998). Furthermore, pairwise population genetic differentiation $\left(\Phi_{\mathrm{pt}}\right)$ was calculated as well as geographic distance used to perform the Mantel test for isolation by distance implemented in the GENALEX 6.3 program (Peakall and Smouse, 2006). The pairwise geographic distances were calculated in kilometers using the latitude/longitude coordinates of each sampled location. Gene flow was calculated using Wright's formula: $N_{\mathrm{m}}=0.25\left(1-F_{\mathrm{ST}} / F_{\mathrm{ST}}\right)(\mathrm{Nei}, 1987)$ and adjusted for diploid data where $M=2 N_{\mathrm{m}}$, with $M$ the number of migrants per generation, $N$ the effective population size and $m$ the migration rate. The STRUCTURE 2.2 program (Pritchard et al., 2000; Falush et al., 2003) was used to investigate the genetic identity of the Houbara populations especially the macqueenii mixed origins. The program implements a Bayesian clustering method to infer and assign the individuals of uncertain origins or without $a$ priori information on their exact geographic origin to populations via their estimated allele frequencies. The analysis were carried out using $\mathrm{K}=2$ to 3 clusters and burns of 25,000 repetitions and 100,000 iteration for Markov chain Monte Carlo (MCMC). Each run was repeated five times to get consistent results.

\section{RESULTS AND DISCUSSION}

\section{Genetic variability within populations}

The Micro-Checker and GENEPOP packages showed no signs for large allele dropout or for scoring errors due to stuttering and no evidence for null alleles. However, the global Fisher test was significant and revealed two genotypic linkage disequilibria between D119 and A113a and between D118 and D110 loci. For this reason, A113a and D110 loci were eliminated from this study. The seven remaining microsatellite loci were successfully amplified in all 10 Houbara populations and generated 1821 genotypes and 55 alleles for a total of 266 individuals. Measures of genetic variability for the 10 populations are shown in Table 1. The mean number of alleles ranged from 2.14 in the Middle East to 6.00 in Errachidia with an average of 4.64. Eight private alleles were found, one in Laayoune, three in Errachidia, two in Iran, one in the macqueenii mixed origins, and one in Afghanistan population. The observed number of alleles reflects historical events of each population, which could include changes in effective population size, impact of natural and artificial selection, introgression, and genetic isolation. Most noticeably is the number of private alleles in the Iranian and Errachidia populations, 2 and 3, respectively. The presence of such private alleles could be due to new accumulated alleles in recently diverged but genetically isolated populations or the result of loss of alleles after bottleneck and divergence. Moreover, the average PIC across populations was calculated to $56 \%$. The Boudnib and Errachidia populations had the highest PIC of $66 \%$ and the Middle East population had the lowest PIC value of $25 \%$. The average PIC value indicated thus that the implemented microsatellite markers were informative and appropriate for such genetic studies (Botstein et al., 1980). The low PIC in the Middle East population is possibly due to the low number of segregating alleles. 


\begin{tabular}{|c|c|c|c|c|c|c|c|c|c|}
\hline Subspecies & Populations & $\mathrm{N}$ & $N_{\mathrm{A}}$ & $\mathrm{PA}$ & PIC & $H_{\mathrm{O}}$ & $H_{\mathrm{E}}$ & HWE & $F_{\text {IS }}$ \\
\hline \multirow[t]{4}{*}{ Chlamydotis undulata } & Erfoud (E. Morocco) & 25 & 4.857 & - & 0.615 & 0.636 & 0.675 & 0.024 & 0.058 \\
\hline & Laayoune (S. Morocco) & 18 & 4.571 & 1 & 0.615 & 0.614 & 0.689 & 0.024 & 0.111 \\
\hline & Errachidia (E. Morocco) & 89 & 6.000 & 3 & 0.666 & 0.625 & 0.717 & 0.000 & 0.127 \\
\hline & Boudnib (E. Morocco) & 21 & 5.142 & - & 0.665 & 0.687 & 0.729 & 0.000 & 0.059 \\
\hline \multirow[t]{5}{*}{ C. u. fuertaventurae } & Fuerteventura (Canary Islands) & 21 & 4.142 & - & 0.542 & 0.582 & 0.618 & 0.269 & 0.059 \\
\hline & Iran & 16 & 5.000 & 2 & 0.574 & 0.548 & 0.633 & 0.000 & 0.138 \\
\hline & Middle East & 5 & 2.142 & - & 0.255 & 0.371 & 0.326 & 0.889 & -0.155 \\
\hline & Baluchistan (Pakistan) & 28 & 5.571 & - & 0.550 & 0.526 & 0.599 & 0.005 & 0.123 \\
\hline & Afghanistan & 6 & 3.857 & 1 & 0.527 & 0.547 & 0.634 & 0.060 & 0.148 \\
\hline C. u. macqueenii & $\begin{array}{l}\text { macqueenii mixed origins } \\
\text { (Kazakhstan, Pakistan, Saudi Arabia) }\end{array}$ & 37 & 5.142 & 1 & 0.587 & 0.590 & 0.652 & 0.124 & 0.094 \\
\hline Mean & & - & 4.642 & - & 0.560 & 0.573 & 0.627 & - & - \\
\hline
\end{tabular}

The values of the $H_{\mathrm{O}}$ and $H_{\mathrm{E}}$ under HWE within each population were also presented. $H_{\mathrm{O}}$ values ranged from 0.37 in Middle East to 0.68 in Boudnib with an average of 0.57 and $H_{\mathrm{E}}$ varied from 0.32 in the Middle East to 0.72 in Boudnib with an average of 0.62. The $H_{\mathrm{O}}$ within each population except for the Middle East one was lower than the $H_{\mathrm{E}}$. Additionally, the $F_{\text {IS }}$ value ranged from -0.15 in the Middle East population to 0.14 in the Afghan population and the $F_{\text {IS }}$ for all populations except the Middle East population was positive indicating a significant deficit of heterozygotes owing to inbreeding $\left(F_{\text {IS }}>0\right)$, samples from sub-populations pooled together (Wahlund effect), and/or genetic drift. However, the negative $F_{\text {IS }}$ value for the Middle East population could be due to by an asymmetrical sex migration that produced an outbreeding effect in the progeny.

Finally, considering the heterozygote deficit as the alternative assumption, six departures from the HWE were detected by the Fisher global test across populations. Indeed, significant multilocus deviations were found within Errachidia, Boudnib, Iran, Baluchistan, Laayoune, and Erfoud populations at the $\mathrm{P}<0.05$ level. Unfortunately, only few Houbara studies that used SSRs are available for comparison. Lesobre et al. (2010) have studied the genetic characteristics of $\mathrm{Cu}$ in North Africa using the same SSR loci. Their estimates of the mean $H_{\mathrm{E}}, H_{\mathrm{O}}$ and $F_{\text {IS }}$ were $0.70,0.64$ and 0.08 , respectively, for Moroccan populations. In the current study, we estimated $H_{\mathrm{E}}, H_{\mathrm{O}}$ and $F_{\mathrm{IS}}$ to be $0.70,0.65$ and 0.05 , respectively, for the populations we sampled in Morocco, which were mostly similar to the estimates of Lesobre et al. (2010).

\section{Genetic variability among populations}

The structure of subdivided populations is often estimated by F-statistics (Wright 1951). The F-statistics $\left(F_{\mathrm{IS}}, F_{\mathrm{IT}}\right.$ and $\left.F_{\mathrm{ST}}\right)$ of the 7 loci are presented in Table 2 . The $F_{\text {IS }}$ values per locus varied from 0.06 to 0.12 with a mean of 0.10 . The $F_{\mathrm{ST}}$ estimates of population differentiation ranged from 0.09 to 0.13 showing an average $F_{\mathrm{ST}}$ of 0.11 . As presented in Table 3 , estimates of genetic differentiation between pairs of populations and their $\mathrm{P}$ values were significant in the majority of combinations except within Moroccan populations (Boundnib, 
Errachidia, Erfoud, and Laayoune). Moreover, our $F_{\mathrm{ST}}$ estimate for Cum populations (0.067) was slightly higher than that reported (0.0419) by Pitra et al. (2004) who studied the Cum population history where the links between wintering and breeding areas were already defined. Our estimate of the global $F_{\mathrm{ST}}$ in the four Moroccan populations $(0.006)$ was, however, similar to the one reported by Lesobre et al. (2010).

\begin{tabular}{|c|c|c|c|c|}
\hline Locus & $F_{\text {IS }}$ & $F_{\mathrm{IT}}$ & $F_{\mathrm{ST}}$ & $N_{\mathrm{m}}$ \\
\hline A112 & 0.125 & 0.230 & 0.120 & 1.833 \\
\hline A210 & 0.103 & 0.212 & 0.122 & 1.799 \\
\hline D119 & 0.081 & 0.207 & 0.136 & 1.588 \\
\hline D118 & 0.127 & 0.228 & 0.115 & 1.923 \\
\hline A29 & 0.069 & 0.176 & 0.115 & 1.923 \\
\hline A120 & 0.129 & 0.216 & 0.099 & 2.275 \\
\hline D117 & 0.081 & 0.197 & 0.127 & 1.718 \\
\hline Mean & 0.103 & 0.209 & 0.119 & 1.865 \\
\hline Standard deviation & 0.058 & 0.042 & 0.025 & \\
\hline
\end{tabular}

Table 3. Pairwise $F_{\mathrm{ST}}$ between Houbara bustard populations (below diagonal) with probability of significance provided above the diagonal.

\begin{tabular}{lcccccccccc}
\hline & Erfoud & Boudnib & Laayoune & Errachidia & CI & Iran & Baluchistan & MMO & ME & Afghanistan \\
\hline Erfoud & $*$ & 0.054 & 0.180 & 0.180 & 0.000 & 0.000 & 0.000 & 0.000 & 0.000 & 0.000 \\
Boudnib & 0.012 & $*$ & 0.351 & 0.819 & 0.000 & 0.000 & 0.000 & 0.000 & 0.000 & 0.000 \\
Laayoune & 0.025 & 0.003 & $*$ & 0.423 & 0.000 & 0.000 & 0.000 & 0.000 & 0.000 & 0.000 \\
Errachidia & 0.011 & 0.000 & 0.002 & $*$ & 0.000 & 0.000 & 0.000 & 0.000 & 0.000 & 0.000 \\
CI & 0.147 & 0.101 & 0.158 & 0.108 & $*$ & 0.000 & 0.000 & 0.000 & 0.000 & 0.000 \\
Iran & 0.176 & 0.158 & 0.156 & 0.153 & 0.246 & $*$ & 0.000 & 0.009 & 0.009 & 0.018 \\
Baluchistan & 0.192 & 0.162 & 0.180 & 0.164 & 0.244 & 0.050 & $*$ & 0.000 & 0.000 & 0.000 \\
MMO & 0.160 & 0.126 & 0.133 & 0.130 & 0.172 & 0.031 & 0.026 & $*$ & 0.000 & 0.018 \\
ME & 0.348 & 0.298 & 0.335 & 0.304 & 0.443 & 0.186 & 0.201 & 0.208 & $*$ & 0.009 \\
Afghanistan & 0.198 & 0.154 & 0.183 & 0.161 & 0.199 & 0.071 & 0.090 & 0.063 & 0.216 & $*$ \\
\hline
\end{tabular}

$\mathrm{CI}=$ Canary Islands; $\mathrm{MMO}=$ macqueenii mixed origins; $\mathrm{ME}=$ Middle East populations .

To analyze hierarchical molecular variance using AMOVA, we first grouped all populations in one set to get a global idea about the genetic structuring and differentiation among and within populations (Table 4). A significant structuring among all sampled populations was found $\left(F_{\mathrm{ST}}=0.12, \mathrm{P}\right.$ value $\left.=0.000\right)$ with $87.97 \%$ of molecular variance within populations and only $12.03 \%$ among groups. The most probable population groupings and configurations according to four geographic groups where also investigated. Generally, the grouping that exhibits the highest value of among group variation $\left(F_{\mathrm{CT}}\right)$ and is significant indicates the most parsimonious geographic subdivision (Paulo et al., 2002). The $[\mathrm{Cuu}]+[\mathrm{Cuf}]+[\mathrm{Cm}]+[\mathrm{Cm}$ Middle-east $]$ grouping displayed the highest statistically significant value of among group variation with an $F_{\text {CT }}$ value of $0.145(\mathrm{P}=0.000)$. This finding affirms, and is in perfect concordance with, the results shown in Table 3 especially regarding the population of Macqueen's mixed origins and that from the Middle East region. 
A. Korrida et al.

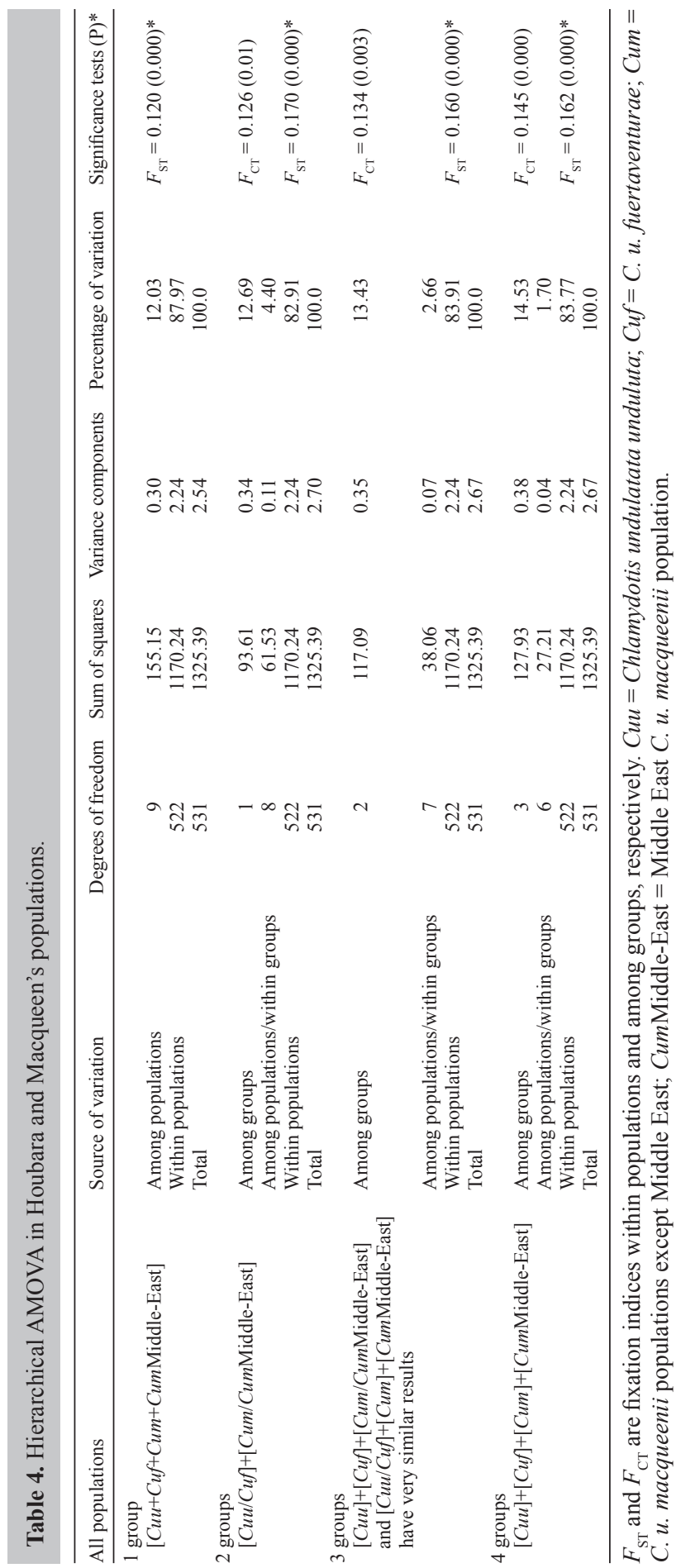


To quantify the sub-structuring for Moroccan Houbara between the South-western and North-eastern populations, we repeated the analysis using separate data for the two groups, which showed an absence of structuring $\left(F_{\mathrm{ST}}=0.0021, \mathrm{P}=0.128\right)$. This estimate corresponded to a number of 238 migrants that were exchanged per generation. Actually, the result was not unexpected as the Houbara have the capacity to disperse over a wide geographic range in Morocco (Idaghdour et al., 2004). Lesobre et al. (2010) also noted that $\mathrm{Cuu}$ founders from Algeria and Eastern Morocco are bred and managed as a single population and their descendants were used to restock wild populations in south eastern Morocco. Furthermore, gene flow within Morocco was reported to be female-mediated, and its considerable levels were sufficient to genetically homogenize the Moroccan populations (Idaghdour et al., 2004). Using the same approach, an estimate of $F_{\mathrm{ST}}=0.1117$ was found between $C u u$ and $C u f$. This Canary Islands Cuf differentiation from the Moroccan $\mathrm{Cuu}$ is due to geographic isolation (sea barrier).

Population structure was further investigated using the STRUCTURE Bayesian clustering method in order to assign individuals to populations based on their genotypes. The most relevant number of clusters was revealed to be $K=2$ as shown in Figure 2. This result was supported by the estimated log probability of the data (LnP(D); Figure 3$)$ and the assessment of the membership percentage to particular clusters (Table 5). The LnP(D) increased by $9.75 \%$ between $\mathrm{K}=1$ and $\mathrm{K}=2$, remained almost constant between $\mathrm{K}=2$ and $\mathrm{K}=3$ and

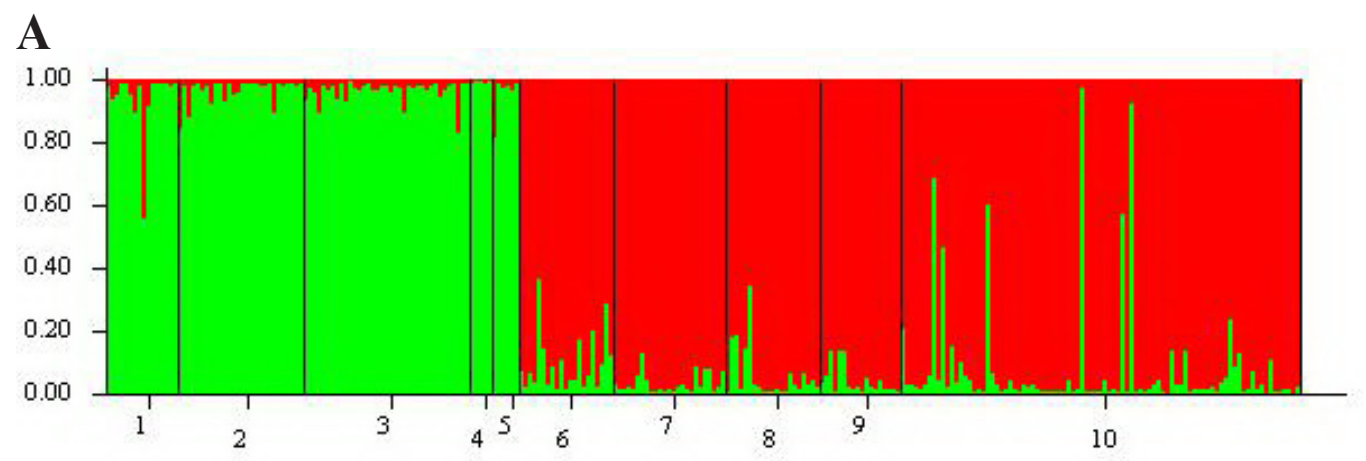

\section{B}

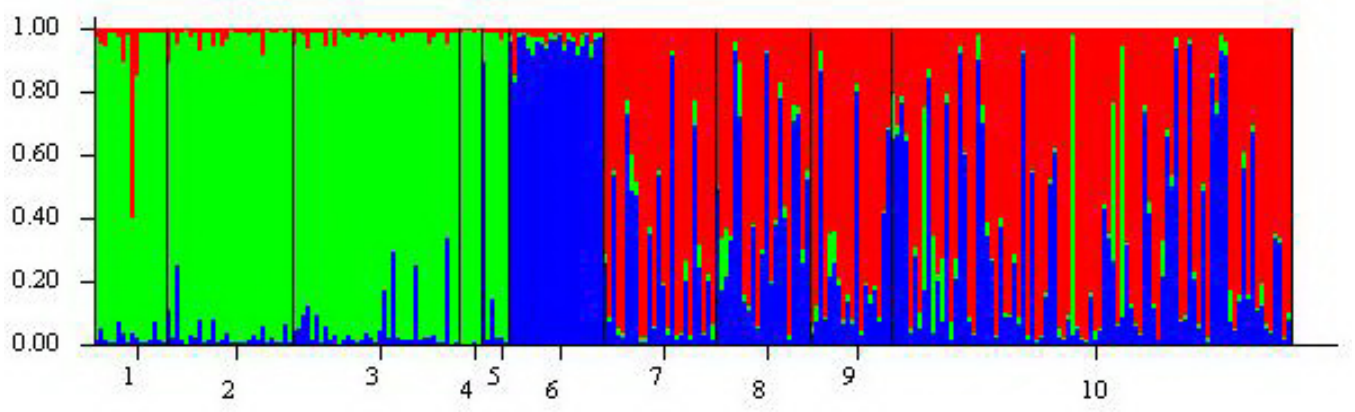

Figure 2. Houbara Bayesian clustering for $\mathrm{K}=2$ and $\mathrm{K}=3$ (with its $\mathbf{A}$ and $\mathbf{B}$ plots). The thin vertical colored bars refer to the proportion of membership to the North African cluster (in red and blue) or to the Asian cluster (in green). 1: Iran; 2: Baluchistan; 3: Macqueenii mixed origins; 4: Middle East; 5: Afghanistan; 6: Canary Islands; 7: Erfoud; 8: Boudnib; 9: Laayoune; 10: Errachidia. 
increased again by $4.72 \%$ between $\mathrm{K}=3$ and $\mathrm{K}=4$ clusters. The weak variation between $\mathrm{K}$ $=2$ and $\mathrm{K}=3$ refers to the plateau reached at $\mathrm{K}=2$ and showed a good fit to the data. This finding is also confirmed by the individual assignment proportion of more than $92 \%$ to either cluster I or cluster II. When the number of clusters was increased to $\mathrm{K}=3$, the admixed haplotypes showed lower percentages of membership with values less than $70 \%$ especially for Moroccan populations.

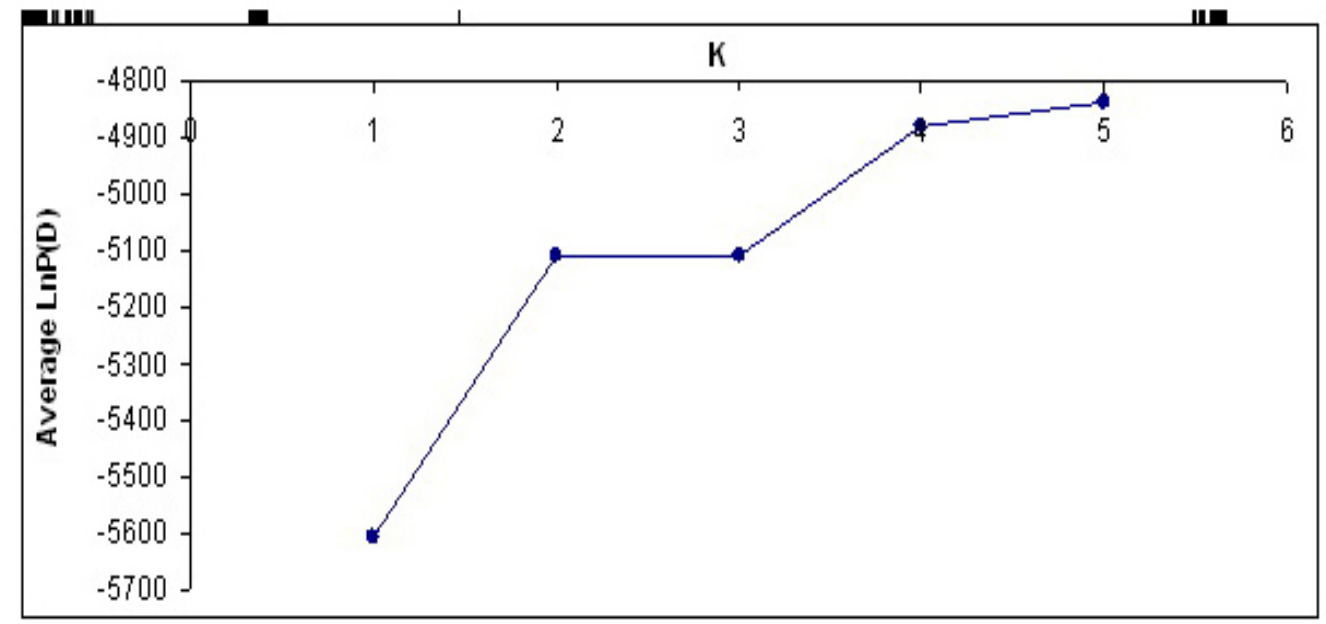

Figure 3. The average posterior probability [log probability of the data, $\operatorname{LnP}(\mathrm{D})]$ of the five runs at each cluster K.

Table 5. Proportion of membership of each pre-defined population to the two major inferred clusters.

\begin{tabular}{llcr}
\hline Subspecies & Populations & Cluster I (\%) & Cluster II (\%) \\
\hline Cum & Iran & 94.50 & 5.50 \\
& Baluchistan & 96.90 & 3.10 \\
& macqueenii mixed origins & 96.80 & 3.20 \\
& Middle East & 99.20 & 0.80 \\
Cuf & Afghanistan & 95.50 & 4.50 \\
Cuu & Canary Islands & 9.40 & 90.60 \\
& Erfoud & 3.50 & 96.50 \\
& Boudnib & 5.90 & 94.10 \\
& Laayoune & 4.30 & 95.70 \\
\hline
\end{tabular}

Cum $=$ Chlamydotis undulata macqueenii $;$ Cuf $=$ C. u. fuertaventurae $;$ Cuu $=$ C. u. undulata.

\section{Genetic distance and gene flow}

A phylogeny tree was drawn using the ME approach (Figure 4). The dendrogram grouped the majority of Houbara and Macqueen's populations into two main clusters $\mathrm{Cuu} / \mathrm{Cuf}$ and Cum and corroborated then the reciprocal monophyly of the Houbara and Macqueen's 
bustards. Also, the ME tree has shown that the North African populations are monophyletic, which confirms further the genetic structuring found between $\mathrm{Cuu}$ and $\mathrm{Cuf}$.

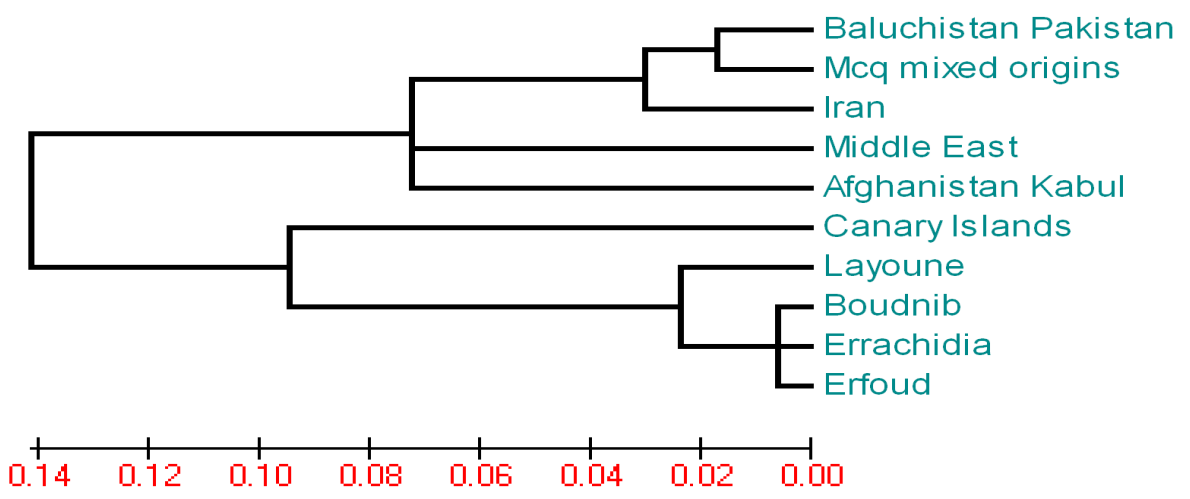

Figure 4. Minimum evolution tree showing grouping of the 10 populations of Houbara and Macqueen's bustards.

In Figure 5 and Table 6, Mantel tests for association between genetic distance, represented by $\left(\Phi_{\mathrm{pt}}\right)$ and geographic distance, revealed that geographic distance plays an important role in the distribution of genetic variation among Houbara populations and showed significant and positive correlations with $\mathrm{r}^{2}=0.302$ and $\mathrm{r}^{2}=0.334$ for the set grouping all populations and the set gathering all population except the one from the Canary Islands, respectively. These genetic findings could be explained by limited genetic connectivity so by relatively high indication of isolation-by-distance between populations. The relationship between genetic differentiation and geographic distance became higher when the $C u f$ population was retrieved from Mantel tests. The decrease in isolation by distance in the first set containing all populations could be explained by the fact that in finite populations such as $\mathrm{Cuf}$, the effect of genetic drift is stronger than gene flow at the studied nuclear loci.

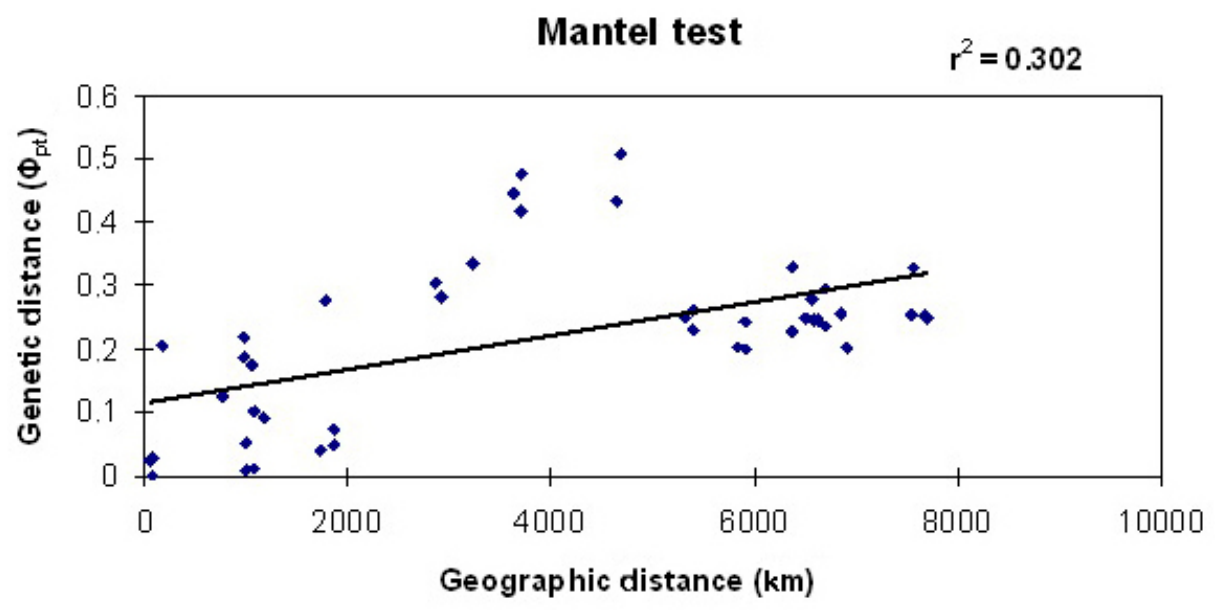

Figure 5. Mantel test for association between genetic distance and geographic distance. 


\begin{tabular}{|c|c|c|}
\hline Popoulations & $\mathrm{r}^{2}$ & $\mathrm{P}$ \\
\hline All (Cuu, Cuf and Cum) & 0.302 & $0.030^{*}$ \\
\hline All except $C u f$ & 0.334 & $0.040^{*}$ \\
\hline All except $C u u$ & 0.355 & 0.113 \\
\hline All except Cum & 0.070 & 0.250 \\
\hline All Cum & 0.543 & 0.220 \\
\hline All Cum except Middle East & 0.836 & 0.100 \\
\hline All Cuи & 0.049 & 0.420 \\
\hline
\end{tabular}

$\mathrm{r}^{2}$ is the coefficient of correlation between population genetic distance and population genetic differentiation $\left(\Phi_{\mathrm{pt}}\right)$. $* \mathrm{P}<5 \%$. For abbreviations, see legend to Table 5 .

Furthermore, the mean $N_{\mathrm{m}}$ value was estimated to 1.86 migrant per generation and historical gene flow between $\mathrm{Cuu}$ and $\mathrm{Cuf}$, Cum and $\mathrm{Cuu}$, and $\mathrm{Cuf}$ and $\mathrm{Cum}$ was $N_{\mathrm{m}}=4.02$ $\left(F_{\mathrm{ST}}=0.111\right), 2.93\left(F_{\mathrm{ST}}=0.145\right)$, and $1.94\left(F_{\mathrm{ST}}=0.204\right)$, respectively. Using mtDNA markers, Idaghdour et al. (2004) showed that gene flow between $\mathrm{Cuu}$ and $\mathrm{Cuf}$ is equal to 1.3 migrants per generation suggesting that males possibly disperse more than females. In fact, the sexbiased dispersal discrepancy is due to larger susceptibility of the female-inherited mtDNA to genetic drift compared to the biparentally inherited microsatellites especially in isolated or differentiated populations (Chesser and Backer, 1996). Idaghdour et al. (2004) reported also that the gene flow is almost exclusively unidirectional from $\mathrm{Cuu}$ to $\mathrm{Cuf}$ as expected given the effective population size of each population.

\section{Scenarios of population differentiation}

The significant differentiation between $\mathrm{Cum}$ and $\mathrm{Cuu}$ reflected by relatively high $F_{\mathrm{ST}}$ value suggests that $\mathrm{Cum}$ split from $\mathrm{Cuu}$ first, then $\mathrm{Cuu}$ and $\mathrm{Cuf}$ became differentiated. This is in concordance with the results of Idaghdour et al. (2004) and Lesobre et al. (2010).

Based on the results generated from this study, we think that the differentiation mechanism within $C$. undulata species could have occurred at two stages. The first between Cum and $\mathrm{Cu} u$ and the second between $\mathrm{Cu} u$ and $C u f$. Assuming that the origin of Otididae family is thought to be in eastern or southern Africa (Pitra et al., 2002), a first hypothesis of C. undulata speciation is an expansion of an ancestral form from eastern Africa to Asia followed by a range expansion all the way to the Atlantic coast of Africa. The second possible hypothesis is that the expansion of the ancestral form occurred at the same time to Asia and North Africa and these two forms were sympatric in Egypt and later separated. Also, the reduction of population size as a result of habitat destruction, excessive hunting and wars (especially that Egypt desert, Middle East and Western-central Asia in Afghanistan, Pakistan, Iran, and Iraq had known long periods of disastrous wars for decades) may be responsible for the currently observed disjunction at the level of Egypt, so in other terms, responsible for the lack of connectivity and isolation resulting in loss of habitat and population fragmentation. Further study on Houbara and Macqueen's mtDNA genes is in progress (Korrida A and Schweizer M, unpublished results) and would shed more light on the two assumptions mentioned above.

For the North African subspecies and according to our measures of microsatellite genetic variability, the most likely mechanism of differentiation between $\mathrm{Cuu}$ and $\mathrm{Cuf}$ is a recent colonization of the Canary Islands. This mechanism agrees also with results previously documented using d-loop marker (Idaghdour et al., 2004). Several models have been developed to 
describe the effects of colonization on the genetic structure of natural populations (Kimura and Weiss, 1964). With regards to the observations related to the low genetic variability in the Canarian subspecies, the most appropriate colonization model might be the stepping-stone model, which assumes that migration occurs between adjacent populations, and that colonized islands contain only a subset of the genetic information of the source population resulting in the loss of genetic diversity due to founder effects (Nei, 1987).

\section{IMPLICATIONS FOR CONSERVATION}

The Houbara and Macqueen's subspecies constitute the main target of Arab falconry and due to their drastic decline has been the focus of the present study. The novelty of the present study resides in the striking evidence it reports for population genetic structuring within Cum being managed currently as a single genetic stock. Indeed, the Middle East population has shown significant levels of genetic differentiation at microsatellite loci, therefore, this very small population would not enable us to draw a decisive conclusion in relation with the definition of management or conservation units as defined by Vogler and Desalle (1994), Moritz (1994) and Crandall et al. (2000). For this end, more samples from the Negev desert, Egypt, Jordan, and/or Sinai areas need to be investigated.

Moreover, our genetic evidence revealed that the geographically isolated Canarian population is also genetically independent and should therefore be considered either as a separate North African evolutionary significant unit (Crandall et al., 2000) or designatable unit (Green, 2005).

The Houbara bustard is a valuable avian resource with great interest for Morocco too. As the four Moroccan populations were found to be very exchangeable with each other, we recommend treating the North-eastern and South-western Moroccan populations as a single management pool. However, similar genetic study should be conducted each time and new samples collected from other Moroccan zones are to be recruited within the captive breeding stocks.

From a wildlife and conservation perspective, we do believe that the current information and results would constitute a reference point and source of knowledge for implementing genetic strategies that could assist in maintaining Houbara bustards' genetic diversity such that the evolvability of population is not at risk, and therefore not compromising chances of adaptation to wild conditions and minimizing the loss of genetic diversity and inbreeding.

Finally, considering that in small and finite populations the risk of extinction within a short time is increased due to over-exploitation, hunting pressure, habitat destruction, pollution, introduced species, and other environmental, genetic and stochastic factors, it is important to mention that beside the information on population structure and heterozygosity estimates held by the neutral genetic markers, direct censuses of the populations could hold more information regarding extinction risk.

\section{ACKNOWLEDGMENTS}

We are grateful to the late HRH Prince Sultan Bin Abdul Aziz Al Saud for his support and for promoting scientific research in Morocco. Thanks are also due to HE Sheikh Mohammed Bin Khalid Bin Hethlein for his encouragements. The authors acknowledge the help of the National Wildlife Research Center at Taif, Kingdom of Saudi Arabia and HE Sheikh Butti Maktoum's 
Wildlife Center at the United Arab Emirates for providing Asian Houbara samples. We extend our thanks also to Oliva Breeding Station in Canary Islands for providing Canarian samples. Special thanks go to the anonymous referees of this manuscript, as well as to Dr. Y. Idaghdour from the Centre de Recherche Sainte-Justine, Université de Montréal for their valuable comments on the manuscript. For their help and assistance, we are also indebted to Pr. S. Benhissoune and Pr. Y. Ait Ichou from Agadir Faculty of Sciences, Ibn Zohr University, B. Iazza from the University of Seville, M.T. Moutaouafiq from Laval University, M.N. Tamssouri and J. Abououalid from Agadir Faculty of Sciences.

\section{REFERENCES}

Belkhir K, Borsa P, Chikhi L, Raufaste N, et al. (2003). GENETIX 4.04, Logiciel Sous Windows ${ }^{\mathrm{TM}}$ Pour la Génétique des Populations. Laboratoire Génome, Populations, Interactions, Adaptations, Umr5171. Université de Montpellier 2, Montpellier.

Birdlife International (2010). Available at [http://www.birdlife.org/]. Accessed August 21, 2010.

Botstein D, White RL, Skolnick M and Davis RW (1980). Construction of a genetic linkage map in man using restriction fragment length polymorphisms. Am. J. Hum. Genet. 32: 314-331.

Chbel F, Broderick D, Idaghdour Y, Korrida A, et al. (2002). Characterization of 22 microsatellites loci from the endangered Houbara bustard (Chlamydotis undulata undulata). Mol. Ecol. Notes 2: 484-487.

Chesser RK and Baker RJ (1996). Effective sizes and dynamics of uniparentally inherited genes. Genetics 144: $1225-1235$.

Cramp S and Simmons KEL (1980). The Birds of the Western Palearctic. Vol. II. Oxford University Press, New York.

Crandall KA, Bininda-Emonds OR, Mace GM and Wayne RK (2000). Considering evolutionary processes in conservation biology. Trends Ecol. Evol. 15: 290-295.

Dawson RJG, Idaghdour Y, Korrida A and Jones C (1999). Molecular genetic analysis of the Houbara bustard populations. IFCDW Bi-Annual Rep. 2: 52-55.

Excoffier L, Lavale G and Schneider S (2006). Arlequin Ver. 3.1: A Software for Population Genetic Analysis. Genetics and Biometry Laboratory, University of Geneva, Geneva.

Falush D, Stephens M and Pritchard JK (2003). Inference of population structure using multilocus genotype data: linked loci and correlated allele frequencies. Genetics 164: 1567-1587.

Gray JE (1832). Otis macqueenii. Hardwicke's Illustrator. Indian Zool. 2.

Green DM (2005). Designatable units for status assessment of endangered species. Conserv. Biol. 19: 1813-1820.

Haddane B (1985). The Houbara bustard in Morocco: a brief review. Bustard Stud. 3: 109-110.

Heim de Balsac H and Mayaud N (1962). Les Oiseaux du Nord-Ouest de l'Afrique. Paris.

Idaghdour Y, Broderick D, Korrida A and Chbel F (2004). Mitochondrial control region diversity of the Houbara bustard Chlamydotis undulata complex and genetic structure along the Atlantic seaboard of North Africa. Mol. Ecol. 13: 43-54.

IUCN (2010). The International Union for Conservation of Nature. Available at [http://www.iucnredlist.org/]. Accessed May 25, 2010.

Jacquin (1784). Psophia undulata. Beytr Gesch Vogel 24, Plate 9.

Keller LF, Arcese P, Smith JN, Hochachka WM, et al. (1994). Selection against inbred song sparrows during a natural population bottleneck. Nature 372: 356-357.

Kimura M and Weiss GH (1964). The stepping stone model of population structure and the decrease of genetic correlation with distance. Genetics 49: 561-576.

Knox AG, Collinson M, Helbig AJ, Parkin DT, et al. (2002). Taxonomic recommendations for British birds. Ibis 144: 707-710.

Lesobre L, Lacroix F, Caizergues A, Hingrat Y, et al. (2010). Conservation genetics of Houbara Bustard (Chlamydotis undulata undulata): population structure and its implications for the reinforcement of wild populations. Conserv. Genet. 4: 1489-1497.

Lewis PO and Zaykin D (2001). Genetic Data Analysis: Computer Program for the Analysis of Allelic Data. Version 1.0. Marshall TC, Slate J, Kruuk LEB and Pemberton JM (1998). Statistical confidence for likelihood-based paternity inference in natural populations. Mol. Ecol. 7: 639-655.

Moritz C (1994). Defining “evolutionary significant units” for conservation. Trends Ecol. Evol. 9: 373-375.

Nei M (1987). Molecular Evolutionary Genetics. Columbia University Press, New York.

Genetics and Molecular Research 11 (3): 3207-3221 (2012)

CFUNPEC-RP www.funpecrp.com.br 
Nei M, Kumar S and Takahashi K (1998). The optimization principle in phylogenetic analysis tends to give incorrect topologies when the number of nucleotides or amino acids used is small. Proc. Nat. Acad. Sci. 95: 12390-12397.

Paulo OS, Jordan WC, Bruford MW and Nichols RA (2002). Using nested clade analysis to assess the history of colonization and the persistence of populations of an Iberian Lizard. Mol. Ecol. 11: 809-819.

Peakall R and Smouse PE (2006). GENALEX 6: genetic analysis in Excel. Population genetic software for teaching and research. Mol. Ecol. Notes 6: 288-295.

Pitra C, Lieckfeldt D, Frahnert S and Fickel J (2002). Phylogenetic relationships and ancestral areas of the bustards (Gruiformes: Otididae), inferred from mitochondrial DNA and nuclear intron sequences. Mol. Phylogenet. Evol. 23: 63-74.

Pitra C, D'Aloia MA, Lieckfeldt D and Combreau O (2004). Genetic variation across the current range of the Asian Houbara bustard (Chlamydotis undulata macqueenii). Conserv. Genet. 5: 205-215.

Pritchard JK, Stephens M and Donnelly P (2000). Inference of population structure using multilocus genotype data. Genetics 155: 945-959.

Raymond $\mathrm{M}$ and Rousset $\mathrm{F}$ (1995). GENEPOP (version 1.2): population genetics software for exact tests and ecumenicism. J. Hered. 86: 248-249.

Rice WR (1989). Analyzing tables of statistical tests. Evolution 43: 223-225.

Rothschild W and Hartert E (1894). On a new bustard from the Paleartic region. Nov. Zool. 1: 689.

Sambrook J, Fritsch EF and Maniatis T (1989). Molecular Cloning: A Laboratory Manual. Cold Spring Harbor Laboratory Press, Cold Spring Harbor.

Sangster G, Hazevoet CJ, Van den Berg AB, Roselaar CS, et al. (1999). Dutch avifaunal list: Species concepts, taxonomic instability, and taxonomic changes in 1997-1998. Ardea 87: 139-165.

Schuelke M (2000). An economic method for the fuorescent labeling of PCR fragments. A poor man's approach to genotyping for research and high-throughput diagnostics. Nat. Biotechnol. 18: 233-234.

Serrano M, Calvo JH, Martinez M, Marcos-Carcavilla A, et al. (2009). Microsatellite based genetic diversity and population structure of the endangered Spanish Guadarrama goat breed. BMC Genet. 10: 61.

Tamura K, Dudley J, Nei M and Kumar S (2007). MEGA4: Molecular evolutionary genetics analysis (MEGA) software version 4.0. Mol. Biol. Evol. 24: 1596-1599.

Van Oosterhout C, Hutchinson WF, Wills DPM and Shipley P (2004). MICRO-CHECKER: software for identifying and correcting genotyping errors in microsatellite data. Mol. Ecol. Notes 4: 535-538.

Vogler AP and Desalle R (1994). Diagnosing units of conservation management. Conserv. Biol. 8: 354-363.

Weir BS and Cockerham CC (1984). Estimating F-statistics for the analysis of population structure. Evolution 38: 13581370.

Wright S (1951). The genetical structure of populations. Ann. Eugen. 15: 323-354. 